\title{
Gianpaolo Chiriacò: Voci nere. Storia e antropologia del canto afroamericano. Milano: Mimesis, 2018. ISBN 978-8857548050. 214 pagine.
}

Appassionato, coinvolgente, necessario. Voci Nere è un libro sulla unicità e, insieme, sulla universalità del canto afroamericano. Ė un libro scritto ad alta voce, come dichiara lo stesso autore (9), Gianpaolo Chiriacò, antropologo della musica, studioso di canto e docente di etnomusicologia presso la Libera Università di Bolzano. Chiriacò propone il suo punto di vista, posizionato all'intersezione tra storia e antropologia, sul tema della vocalità black, ma facendo spazio alle voci degli altri, di tutte le persone incontrate in oltre due anni di ricerca sul campo e in archivio. Tra il 2012 e il 2014 ha lavorato quotidianamente nel Center for Black Music Research di Chicago nell'ambito del progetto Afrovocality, che ha investigato il ruolo degli stili vocali tradizionali nel rimodellare le identità culturali connesse alla diaspora africana in America e in Europa. È da questa intensa esperienza che nasce questo libro, pensato per docenti e studenti di etnomusicologia, popular music studies, cultural studies, canto jazz e pop, ma fruibile da qualsiasi lettore interessato al tema del ruolo 'sociale' della voce nella cultura afroamericana.

È un libro scritto ad alta voce anche per la sua narrazione "transmediale" (Jenkins, 2007), grazie agli efficaci inserti di $Q R$-Code ${ }^{1}$, che rimandano ai contenuti audiovisivi a cui si fa rifermento nel testo (tutti disponibili nella sezione "Multimedia" del sito del progetto di ricerca $w w w$.afrovocality.com), offrendo una lettura piacevolmente dinamica.

Il volume è strutturato in due parti: nella prima, "Un'oralità imposta", la prospettiva è prevalentemente di tipo storico, mentre nella seconda parte, "Dall'era post-razziale al trauma Trump", emerge la prospettiva antropologica. I due punti di vista, tuttavia, si intersecano continuamente, data la natura interdisciplinare del discorso sulla voce, che lascia da parte qualsiasi approccio lineare o percorso unidirezionale. Già dalle prime pagine emerge che "la storia di una voce è qui anche la voce di una storia" (12). Nel primo capitolo, infatti, Chiriacò si sofferma sul concetto di "memorie loquaci": la tradizione orale afroamericana cancella lo scarto temporale tra il passato degli ancestors (gli antenati, che vengono definiti, secondo il concetto coniato dalla studiosa Farah Jasmine Griffin, "talkative", loquaci) e il presente del performer che non rappresenta mai solo se stesso, ma incarna una continuità identitaria. Questo concetto fondamentale viene efficacemente spiegato al lettore raccontando l'esibizione di Tammy McCann che, in una serata a Chicago nel 2014 dedicata a Nina Simone, sceglie di interpretare il brano "Black Is the Colour of My True Love's Hair", un canto popolare tradizionale originario degli Appalachi ed entrato a far parte del repertorio 
di diversi generi musicali, ma che in quella occasione racchiudeva tutto il suo modo di essere black (19-21).

Chiriacò avvia nel secondo capitolo una lunga riflessione su quanto la tradizione orale afroamericana sia indissolubilmente legata alla schiavitù; il ruolo della voce, infatti, si afferma in seguito a una imposizione, quella contenuta negli slave codes, che rendevano illegale l'istruzione degli schiavi e punivano con la morte quelli che venivano sorpresi a leggere e scrivere. La chiave di lettura dei capitoli della prima parte del volume è proprio il ruolo della voce nel colmare il vuoto creato dalla soppressione di ogni altra possibilità espressiva, ma anche il suo rapporto, materiale e simbolico, con lo spazio in cui risuonano le voci degli antenati (36). In primo luogo, Chiriacò propone una interessante analisi della descrizione di questo spazio nel film del regista inglese Steve McQueen 12 Years a Slave. Uscito nel 2013, il film racconta con crudezza la vera storia di un nero americano, un violinista, che viveva libero nel Nord degli Stati Uniti e venne rapito e tradotto in schiavitù in Louisiana nel 1841. Particolarmente importante per il lavoro di Chiriacò è l'osservazione di come il rapporto tra voce e silenzio costituisca un elemento fondamentale per definire una idea di blackness, nonché una delle strategie di sopravvivenza messe in atto dagli schiavi: il protagonista, nato libero, vorrebbe proclamare la sua non-appartenenza alla slave culture, che si esprime nella voce cantata e a cui egli oppone il silenzio. Tuttavia, si renderà conto che solo cantando potrà dar voce ai suoi sentimenti e preservare la propria identità non solo di "schiavo libero" (47), espressione che suona come un ossimoro, ma di uomo.

Il rapporto, geografico e politico, tra voce e spazio è oggetto di analisi dei due capitoli successivi. Nel terzo capitolo l'autore si occupa della tradizione vocale dei field hollers, richiami vocali utilizzati come strumento di comunicazione durante il lavoro degli schiavi nei campi e considerati anticipatori del blues. Chiriacò ripercorre sinteticamente i lavori di studiosi di vari ambiti teorici: la definizione di nation language coniata da Kamau Brathwaite per riferirsi alla varietà non-standard della lingua inglese nelle comunità nere caraibiche, l'analisi di Paul Zumthor sulla "presenza della voce" del performer in un determinato spazio fisico, l'attenzione alla dimensione relazionale dell'ascolto di Jean-Luc Nancy, il potere sacralizzante della voce studiato da Walter Ong, fino al contributo filosofico e musicologico di Carlo Serra sulla voce cantata come "gesto sonoro", quindi movimento, ed "evocazione sonora" della memoria di una comunità (56). Entrambi questi elementi sono ritenuti da Chiriacò cruciali per capire l'essenza e l'enorme potere simbolico e comunicativo dei field hollers, la cui principale funzione era "la riformulazione delle relazioni spaziali in tre direzioni diverse: con l'ambiente acustico, con gli altri esseri umani, e con un'evocazione simbolica” (69). Nel quarto capitolo, invece, l'autore propone una interpretazione delle memorie di canto nelle ex-slave interviews. L'ampio inquadramento teorico di riferimento - a partire dal testo del 1928 di Milton Metfessel, Phonophotography in Folk Music, dove vengono classificate le cosiddette black utterances, tratti vocali stilistici peculiari del Negro singing - introduce la presentazione di Freedom Was a Singsong, una installazione a cura dell'autore disponibile online sul sito www.afrovocality.com. In questa accurata voice map, Chiriacò ha ricostruito una "cartografia della liberazione", in cui la stessa libertà diventa singsong, momento di can- 
to, ricordo vocale (80), e lo ha fatto collocando 120 estratti di slave interviews - dove il canto e la voce sono centrali nel ricordo degli ex-schiavi - su una mappa degli Stati Uniti del 1863, anno in cui Lincoln firma la proclamazione della emancipazione dalla schiavitù.

Il quinto capitolo, l'ultimo della prima parte, è dedicato al ruolo dell'iconografia nella rappresentazione della vocalità, con particolare riferimento alla città di Chicago, dove l'autore ha vissuto, e alla figura poliedrica di Theaster Gates, la cui pratica artistica (da lui stesso definita neighborhood) spazia dalla performance alle installazioni urbane ed è associata ad un forte impegno sociale nel South Side, la parte di Chicago che comprende i quartieri più disagiati, non senza qualche critica da parte di chi rimane fortemente scettico nei confronti della gentrificazione, soprattutto per le conseguenze in termini economici che i nuovi investimenti possono avere sugli abitanti originari, che non possono più permettersi di risiedere nei quartieri trasformati per via dell'aumento dei prezzi. Chiriacò si sofferma, poi, sull'intervento di Yaw Agyeman, collaboratore di Gates, nel symposium Black Vocality, di cui si parlerà ampiamente nei capitoli seguenti. In quella occasione Yaw esordisce con un canto - trascritto nel testo e ascoltabile grazie al puntuale $Q R$-Code (92) - che incarna l'estetica blues, concetto proposto negli anni novanta dal critico d'arte Richard Powell e che l'autore riconduce al ruolo della voce, "che passa dai polmoni alle corde vocali allo spazio attorno al corpo nero del cantante, diventa allo stesso tempo performance di blackness e superamento di quei confini identitari" (97).

Che il concetto di estetica blues oltrepassi i confini identitari e che non abbia nulla a che fare con una tecnica specifica viene ribadito nel sesto capitolo, il primo della seconda parte, in cui Chiriacò precisa ancora una volta che la vocalità nera non è in alcun modo in relazione con il colore della pelle (nonostante questa sia opinione diffusa anche tra alcuni musicologi), bensì con un patrimonio di memorie sonore condiviso nella comunità afroamericana. E a questo punto che l'autore, che ha studiato a lungo canto jazz e cantato nel coro Gospel del Conservatorio di Lecce, ritiene opportuno spiegare perché ha preferito non adottare nella sua ricerca una prospettiva da insider, ovvero non fare musica insieme ai vocalist con cui ha lavorato. Chiriacò, consapevole del suo "innegabile privilegio di accademico bianco ed europeo" (13), ha voluto "posizionarsi a una distanza rispettosa” (104), che gli sembrava necessaria per sviluppare un'analisi di come la vocalità afroamericana nasca da un' esperienza storica e culturale in una comunità che ancora oggi deve fare i conti con le disuguaglianze sociali. L'autore dedica il capitolo al suo incontro con Lena McLin, leggendaria docente di canto della Kenwood Academy di Chicago oggi ultranovantenne. Corredato dai puntuali $Q R$-Codes, il suo racconto, emozionato e lucido insieme, fa sentire anche il lettore a casa di questa bellissima signora, che ha sempre insegnato ai suoi studenti, neri e bianchi, soprattutto a essere persone, a essere veri. Nelle sue lezioni, infatti, lo specchio è sempre stato un elemento fondamentale non per adottare una determinata postura sul palcoscenico, ma per prendere consapevolezza del proprio corpo e riconoscere se stessi. E per riconoscere se stessi, per diventare coscienti della propria umanità, l'altro elemento fondamentale del metodo di insegnamento di Lena McLin è la conoscenza della storia, perché quando si canta non si rappresenta mai solo se stessi: "cantare è l'espressione di un sentimento interiore [...] è un 
movimento del pensiero, una disamina della situazione particolare che ha condotto a quel sentimento" (118).

C’è una prevalenza di voci nere femminili nel volume di Chiriacò, che nel settimo capitolo si sofferma su quella di Maggie Brown, figlia di Oscar Brown Jr., artista, intellettuale e attivista per i diritti civili, che nella sua performance all'interno del symposium Black Vocality pone enfasi sullo storytelling e sull'edutainment, la capacità di educare intrattenendo. Maggie Brown incarna la contraddizione di chi ha imparato il mestiere sul campo, "è scappata dal circo", per usare la sua stessa espressione (124) e ora non ha le carte in regola per fare tutto quello che avrebbe potuto fare se solo avesse finito gli studi, ad esempio insegnare musica all'università. L'intervento di Maggie Brown racconta della legacy, l'eredità musicale che le è stata consegnata, con tutti gli onori e gli oneri, e offre un esempio dello stile vocalico dello storytelling afroamericano attraverso l'utilizzo della tonal semantics (Smitherman 1977), espressione che fa riferimento ai significati affettivi ed emotivi veicolati dalla manipolazione del tono, del timbro e del volume della voce, nonché di altri elementi paralinguistici quali la gestualità.

Alcuni necessari dettagli sugli aspetti pratici del progetto di ricerca dell'autore, non senza qualche vicissitudine, sono il tema dell'ottavo capitolo. Con la sua prosa coinvolgente Chiriacò racconta di come l'idea del symposium Black Vocality sia nata dopo una conversazione con Samuel Floyd, fondatore del Center for Black Music Research di Chicago, che proprio nel 2012, anno in cui Chiriacò iniziava la sua ricerca sul campo, ha conosciuto una profonda crisi amministrativa che ne bloccava formalmente le attività. Black Vocality ha in qualche modo colmato questa lacuna, diventando nelle sue due edizioni il luogo dove vocalist, studiosi, letterati, studenti e semplici interessati hanno potuto incontrarsi (141).

Il nono capitolo è dedicato alla straordinaria figura di Nina Simone nel contesto contemporaneo, per capire la quale l'autore espone il concetto di “complicità con gli eccessi” (147): nella società americana, per raggiungere il successo non si può fare a meno di accettare la complicità con un passato e un presente di violenze e disuguaglianze. Questo emerge, tra l'altro, nella sua autobiografia, in cui Nina Simone racconta di come si sia resa conto che una canzone come "Mississippi Goddam", che nasce da una rabbia insopportabile nei confronti di due eventi drammatici verificatisi a spese degli afroamericani all'inizio degli anni sessanta, può essere un'arma molto più efficace di una pistola. Per Chiriacò la singolarità della figura di Nina Simone emerge anche nel suo prendere le distanze dalla tradizione musicale religiosa: in quella stessa canzone l'artista afferma, infatti, di aver smesso di credere alle preghiere. Altro elemento importante è la vicinanza di Nina Simone ad attori comici come Richard Pryor e ad artisti come Langston Hughes e James Baldwin, con i quali non risparmiava battute a sfondo politico che poi si esprimevano in canzoni come "Go Limp", dove una tattica di disobbedienza civile (il non opporre alcuna resistenza fisica al momento dell'arresto per rendere quest'ultimo estremamente complicato) assume connotazioni sessuali (152). Nella seconda parte del capitolo, Chiriacò riporta alcuni passaggi della performance Channeling Nina all'interno del symposium Black Vocality, dove la solidità della voce di Nina Simone ha risuonato come "fatto sonoro" (157), come fenomeno inconfutabile. Emblematiche 
sono le parole di uno dei vocalist partecipanti, Napoleon Maddox, che incontreremo più avanti nel libro e che nel tentativo di spiegare il fascino dell'artista afroamericana elenca una serie di otto parole, che iniziano tutte per P: Passion, Poetry, Protest, Provocation, Political, Pissed off, Purpose, Power (158-159). Per Maddox non si può prescindere da alcuna di queste parole per parlare dell'opera di Nina Simone, ma la chiave di lettura è la parola Purpose, che racchiude il passaggio dalla necessaria "complicità con gli eccessi” alla innegabile e non rinnegabile dimensione relazionale con la comunità afroamericana.

Il rapporto con gli ancestors e il significato di "vocalità nera” tornano nel decimo capitolo, che riporta l'intervista dell'autore con una delle vocalist più attive nel panorama della scena jazz di Chicago, Dee Alexander. Alla domanda di Chiriacò sul significato di vocalità nera, l'artista risponde che per lei i vocalist neri sono soulful (166), termine difficilmente traducibile in italiano, ma questa peculiarità degli artisti neri sembra essere smentita subito dopo, quando afferma che anche tanti altri vocalist di culture diverse sono soulful: a cambiare è solo il modo di esprimersi e di raccontare le proprie storie, e per la comunità afroamericana questa modalità è colourful, piena di colore, come il quilt, la coperta patchwork fatta dalle donne nere con avanzi di stoffa rattoppati, simbolo di quella vera e propria "arte del riuso" che la comunità afroamericana ha sempre fatto propria, dimostrandosi capace di utilizzare tutto quello che si ha a disposizione. Una pratica che per Dee Alexander avviene soprattutto attraverso l'ascolto, prima di tutto del proprio corpo, e che per lei ha una definizione precisa ed efficace: "I take everything and make my own cake." (168)

Nell'undicesimo capitolo Chiriacò riporta i suoi dialoghi con uno dei personaggi più carismatici che si incontrano nel libro, Napoleon Maddox, di cui si è detto prima. Rapper e vocalist originario di Cincinnati, l'artista ha all'attivo prestigiose collaborazioni con alcuni fra i più importanti nomi del jazz internazionale e ha contribuito - con la sua voce, i suoi versi e il suo beatboxing - ai set dei maggiori artisti Hip Hop. A questo si aggiunge il suo instancabile lavoro come attivista: vicino al movimento internazionale Black Lives Matter, attualmente impegnato nella lotta contro il razzismo, Napoleon ha lavorato in molte circostanze come educatore, organizzando workshop per bambini e adolescenti appartenenti a minoranze o provenienti da quartieri a rischio. Insieme a loro utilizza i linguaggi del rap e del beatboxing (alla cui tecnica e al cui potenziale comunicativo il capitolo dedica ampio spazio), nonché la poesia, per fornire loro strumenti espressivi che spesso trovano anche spazio in performance, come nel caso del progetto sviluppato nelle banlieues parigine A Riot Called Nina, dove la figura di Nina Simone è utilizzata per riflettere sulle possibilità di realizzare azioni che abbiano un impatto sulla giustizia sociale. Chiriacò racconta, tra l'altro, della produzione presentata da Maddox a Torino nel 2017, Twice the First Time, che ripropone la vera storia di Millie-Christine McCoy, due gemelle siamesi nate in schiavitù in North Carolina nel 1851 e di cui Napoleon è diretto discendente. Poiché inadatte al lavoro nei campi, il loro padrone decise di utilizzare la loro unicità trasformandole in un fenomeno da baraccone. In deroga agli slave-codes di cui si è detto ampiamente nella prima parte, le sorelle impararono a leggere e a scrivere, a suonare e a cantare, fino a diventare delle vere e proprie star internazionali. Raccontando la storia di Millie-Christine come esempio di 'diversità' e 
successo, ma anche come metafora di identità multiple, Maddox costruisce intorno alla sua voce e al suo corpo nero (ispirandosi al bellissimo libro del 2015 del giornalista Ta-Nehisi Coates, Between the World and $\mathrm{Me}$ ) una performance dall'elevato livello di interdisciplinarità, giustapponendo diversi stili vocali e incoraggiando una profonda riflessione su come sia possibile nella società odierna e soprattutto per i giovani, relazionarsi alla loro storia e parlare senza riserve di razzismo e discriminazioni, in altre parole "abitare il tratto" (179), quello che separa la parola 'africano' dalla parola 'americano' e che Maddox vede come metafora della storia dei neri in America, figlia del colonialismo e della diaspora.

Nel dodicesimo capitolo l'autore torna a parlare di storytelling in contrapposizione a un'altra pratica di oralità moderna, conosciuta con l'espressione spoken word. Se lo storytelling è una pratica più competitiva, dove è la storia a essere al centro della scena, nello spoken word viene messa in primo piano la natura sonica del linguaggio, quindi la vocalità dell'artista. I fondamenti sociali dello spoken word, che per Chiriacò è una pratica di libertà, sono il coming oute il coming together, ovvero il dichiararsi nella propria soggettività facendo in modo che questa venga accettata dal pubblico (185). L'autore racconta di alcuni degli artisti di spoken word incontrati nell'ambito dell'evento Front Porch, curato da Tim'm West, poliedrico personaggio che si inscrive nella "lunga tradizione di contestatori-artisti afroamericani” (187). La sua complessa storia personale di artista, femminista, gay, nero e sieropositivo in un paese come l'America gli ha dato la capacità di trovare, come dice lui stesso, "la poesia nella teoria e la teoria nella poesia" (190), nonché, utilizzando l'efficace metafora di Chiriacò, l'abilità di "surfare una modernità dai confini liquidi” (191), scongiurando il rischio di venirne sommersi.

Il tredicesimo e ultimo capitolo del volume non è un capitolo conclusivo, ma un capitolo di transizione, che racconta il passaggio dalla cosiddetta era post-razziale della presidenza Obama, che in qualche modo permea gli anni della ricerca sul campo di Chiriacò, al 'trauma' Trump che irrompe proprio a conclusione del suo lavoro. Le voci a cui Chiriacò ha dato voce risuonano più che mai in queste ultime pagine, soprattutto a proposito della questione della vocalità e dell'identità afroamericana, un binomio spesso carico di pregiudizi, come si è visto nel sesto capitolo (quello dedicato alla figura di Lena McLin) e dal quale giovani artisti come Pamela $\mathrm{Z}$ prendono le distanze, rivendicando una identità 'americana', senza alcun trattino che segua il prefissoide 'afro', anche in fatto di gusti musicali. Attraverso la voce, però, si può acquisire "una consapevolezza politica delle proprie possibilità espressive" (202), come fa la vocalist Mankwe Ndosi nei suoi workshop, dove spesso ricorre il concetto di healing, un uso terapeutico della voce per rimarginare le ferite collettive di una comunità ancora vittima di disparità economiche e violenza. E negli ultimi anni la recrudescenza degli episodi di police brutality in diverse città degli Stati Uniti è un sintomo inequivocabile che quella dolorosa "linea del colore" di cui parlava DuBois è ancora molto, troppo marcata. L'ultimo $Q R$-Code proposto da Chiriacò riporta il vigoroso spoken word dal titolo "Stand 4 What" del rapper Nick Cannon, che sulle note struggenti dell'inno nazionale americano eseguito al sax dà voce alla protesta silenziosa del campione di football americano Colin Kaepernick, che dal 2016, durante l'esecuzione dell'inno prima di ogni partita, invece di 
posizionarsi sull'attenti ha cominciato a inginocchiarsi, come presa di posizione contro gli abusi della polizia americana nei confronti degli afroamericani. Il gesto ha suscitato le ire di Trump, ed è proprio a lui che Cannon non le manda a dire nel suo spoken word, facendo esplicito riferimento alla terza strofa dello "The Star-Spangled Banner", quella che con i versi "No refuge could save the hireling and slave / From the terror of flight, or the gloom of the grave" rievoca orgogliosamente la violenta rappresaglia dei patrioti americani contro gli schiavi che durante la guerra del 1812-1814 tra Stati Uniti e Gran Bretagna si erano schierati con quest'ultima in cambio della promessa di libertà. Nella voce di Nick Cannon risuonano tutte le voci nere di Chiriacò: "They may have taken the word slave out / but they forgot to remove the slave connotations / from their brains and their mouth." (195)

In quel 'they', che presuppone un 'us' si può ancora leggere la twoness che lacerava $\mathrm{Du}$ Bois: "[...] un Americano, un Negro; due anime, due pensieri, due lotte non conciliate; due ideali contrastanti in un unico corpo scuro" (Du Bois [1903] 2007, 103). Le voci nere di Chiriacò ci dicono che questa dualità lacera ancora gli Stati Uniti, dove il razzismo rimane strutturale, anche dopo otto anni di presidenza Obama. Per Chiriacò "il razzismo altro non è che l'etica del silenzio" (30), e se uno degli intenti espliciti del suo volume è proprio l'articolazione di una proposta di ascolto e di una riflessione antirazzista, l'autore riesce pienamente a realizzarlo e lo fa sempre "alla ricerca di poesia nella teoria e di teoria nella poesia".

\section{Paola ATTOLINO (Salerno)}

\section{Note}

1 Il Quick Response Code è un codice a matrice in grado di memorizzare informazioni (in questo caso rimandi a video, audio e immagini disponibili online) che possono essere rapidamente decodificate dalla videocamera di uno smartphone.

\section{Bibliografia}

Coates, Ta-Nehisi: Between the World and Me. Melbourne: The Text Publishing Company, 2015.

Du Bois, W.E.B.: Le anime del popolo nero [The Souls of Black Folk, 1903]. Trad. R. Russo. Firenze: Le Lettere, 2007.

Jenkins, Henry: Cultura convergente. Milano: Apogeo, 2007.

Smitherman, Geneva: Talkin and Testifyin. The Language of Black America. Boston: Houghton Mifflin, 1977. 